\title{
Universiteit
}

Leiden

The Netherlands

\section{Direct determination of the Tolman length from the bulk pressures of liquid drops via molecular dynamics simulations}

Giessen, A.E. van; Blokhuis, E.M.

\section{Citation}

Giessen, A. E. van, \& Blokhuis, E. M. (2009). Direct determination of the Tolman length from the bulk pressures of liquid drops via molecular dynamics simulations. Journal Of Chemical Physics, 131(16), 164705. doi:10.1063/1.3253685

Version: $\quad$ Not Applicable (or Unknown)

License: $\quad$ Leiden University Non-exclusive license

Downloaded from: https://hdl.handle.net/1887/62396

Note: To cite this publication please use the final published version (if applicable). 


\section{Direct determination of the Tolman length from the bulk pressures of liquid drops via molecular dynamics simulations}

Alan E. van Giessen, and Edgar M. Blokhuis

Citation: The Journal of Chemical Physics 131, 164705 (2009); doi: 10.1063/1.3253685

View online: https://doi.org/10.1063/1.3253685

View Table of Contents: http://aip.scitation.org/toc/jcp/131/16

Published by the American Institute of Physics

\section{Articles you may be interested in}

The Effect of Droplet Size on Surface Tension

The Journal of Chemical Physics 17, 333 (1949); 10.1063/1.1747247

A molecular dynamics study of liquid drops

The Journal of Chemical Physics 81, 530 (1984); 10.1063/1.447358

Curvature dependence of surface free energy of liquid drops and bubbles: A simulation study

The Journal of Chemical Physics 133, 154702 (2010); 10.1063/1.3493464

The Statistical Mechanical Theory of Surface Tension

The Journal of Chemical Physics 17, 338 (1949); 10.1063/1.1747248

Thermodynamic expressions for the Tolman length

The Journal of Chemical Physics 124, 074701 (2006); 10.1063/1.2167642

Tolman length and rigidity constants of the Lennard-Jones fluid

The Journal of Chemical Physics 142, 064706 (2015); 10.1063/1.4907588

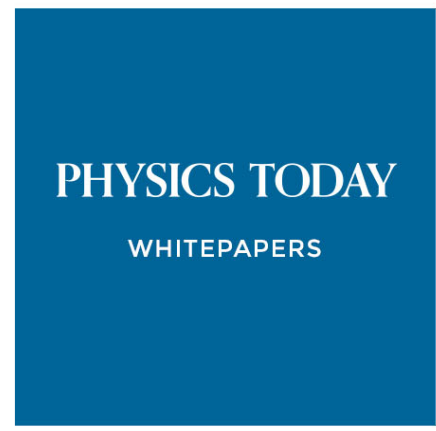

ADVANCED LIGHT CURE ADHESIVES

Take a closer look at what these environmentally friendly adhesive systems can do

\section{READ NOW}

PRESENTED BY Q) MASTERBOND 


\title{
Direct determination of the Tolman length from the bulk pressures of liquid drops via molecular dynamics simulations
}

\author{
Alan E. van Giessen ${ }^{1, a)}$ and Edgar M. Blokhuis ${ }^{2}$ \\ ${ }^{1}$ Department of Chemistry, Hobart and William Smith Colleges, Geneva, New York 14456, USA \\ ${ }^{2}$ Colloid and Interface Science, Leiden Institute of Chemistry, Gorlaeus Laboratories, P.O. Box 9502, \\ 2300 RA Leiden, The Netherlands
}

(Received 9 July 2009; accepted 2 October 2009; published online 30 October 2009)

\begin{abstract}
An expression for the difference in pressure between a liquid drop in equilibrium with its vapor $\Delta p=p_{\ell}-p_{v}$ is derived from previous expressions for the components of the Irving-Kirkwood pressure tensor. This expression, as well as the bulk values of the pressure tensor, is then evaluated via molecular dynamics simulations of particles interacting through a truncated Lennard-Jones potential. We determine the Tolman length $\delta$ from the dependence of $\Delta p$ on the equimolar radius. We determine the Tolman length to be $\delta=-0.10 \pm 0.02$ in units of the particle diameter. This is the first determination of the Tolman length for liquid droplets via the pressure tensor route through computer simulation that is negative, in contrast to all previous results from simulation, but in agreement with results from density functional theory. In addition, we study the planar liquid-vapor interface and observe a dependence of the physical properties of the system on the system size, as measured by the surface area. (C) 2009 American Institute of Physics. [doi:10.1063/1.3253685]
\end{abstract}

\section{INTRODUCTION}

In the study of the surface properties of curved interfaces between coexisting liquid and vapor phases, the quantity of most interest is the surface tension. ${ }^{1}$ It is well known that the surface tension for such interfaces depends on the radius of the liquid droplet or vapor bubble. The pioneering work in this area is by Tolman, ${ }^{2}$ who investigated the dependence of the surface tension of a liquid drop $\sigma(R)$ on the radius $R$. Expanding $\sigma(R)$ in powers of the curvature,

$$
\sigma(R)=\sigma_{0}-\frac{2 \sigma_{0} \delta}{R}+\cdots,
$$

where $\sigma_{0}$ is the surface tension of the planar interface and the quantity $\delta$ is now known as the Tolman length. Such an expansion is complicated by the fact that the interface has a finite width and that there is no unambiguous choice for the location of the interface. For convenience, a mathematical dividing surface $R$ is introduced in the region of the interface. Two choices for this dividing surface are common: the equimolar surface $R_{\mathrm{e}}$, where the excess number density is zero, and the surface of tension $R_{\mathrm{s}}$, where the surface tension is said to "act," and which makes the Laplace equation exact. It can be shown for such an expansion that $\delta$ is independent of the choice of dividing surface. Furthermore, it has also been shown that the Tolman length can be found from the planar limit of the difference between these two dividing surfaces,

$$
\delta=\lim _{R_{\mathrm{e}}, R_{\mathrm{s}} \rightarrow \infty}=z_{\mathrm{e}}-z_{\mathrm{s}} .
$$

The Tolman length plays an important role whenever curved interfaces are present, such as the nucleation of drop-

${ }^{a)}$ Electronic mail: vangiessen@hws.edu. lets saturated vapor. However, while the magnitude of $\delta$ for a simple liquid-vapor interface is generally agreed upon, there is not yet a consensus upon the sign of $\delta .^{3}$ Theoretical estimates using density functional theory (DFT) result in negative values for the Tolman length, ${ }^{4-8}$ while computer simulations of planar interfaces give positive values for the Tolman length, via Eq. (6) below, ${ }^{9-11}$ or were inconclusive. ${ }^{12,13}$ Moody and Attard ${ }^{14}$ determined the Tolman length from simulations of a liquid near coexistence solvating a hard-wall cavity and found that the Tolman length was positive at low temperatures, changed sign at a certain temperature, and became increasingly negative as the temperature increased. It is not clear how the Tolman length for a solvated hard-wall cavity in a liquid near coexistence compares with that of a liquid drop surrounded by its vapor, though these authors give reasons why they ought to be the similar. A negative Tolman length has also been reported for simulations based on a cluster model. ${ }^{15}$

The expressions used to determine the Tolman length in these simulations are derived from the Laplace equation. Classically, this is given as ${ }^{1}$

$$
\Delta p=\frac{2 \sigma_{\mathrm{s}}}{R_{\mathrm{s}}},
$$

and relates the ratio of the surface tension of a droplet to the surface of tension $R_{\mathrm{s}}$ to $\Delta p \equiv p_{\ell}-p_{v}$, the difference between pressures of the bulk liquid and vapor phases. Writing this in terms of the equimolar radius $R_{\mathrm{e}}$ allows an expansion in powers of the curvature

$$
\Delta p=\frac{2 \sigma_{0}}{R_{\mathrm{e}}}\left(1-\frac{\delta}{R_{\mathrm{e}}}+\cdots\right) .
$$

The natural use of Eq. (4) is to determine the dependence of $\Delta p$ on the curvature, and therefore determine the values of 
both the surface tension of the planar interface and the Tolman length. Such an approach has been attempted before, ${ }^{12,13}$ with limited success due to the difficulty of the calculation and the limited computational resources available at the time. Instead, Eq. (4) has been used to derive the so-called "virial" expressions for both the surface tension of a planar interface and for the Tolman length. 9,16 The surface tension is given by

$$
\sigma_{0}=\frac{1}{4} \int \mathrm{d} z_{1} \int \mathrm{d} \mathbf{r}_{12} u^{\prime}(r) r\left(1-3 s^{2}\right) \rho_{0}^{(2)}\left(z_{1}, z_{2}, r\right),
$$

while the Tolman length is given by either of two equivalent expressions,

$$
\begin{aligned}
\delta= & -\frac{1}{8 \sigma_{0}} \int \mathrm{d} z_{1} \int \mathrm{d} \mathbf{r}_{12} u^{\prime}(r) r\left(1-3 s^{2}\right)\left(z_{1}+z_{2}\right) \\
& \times \rho_{0}^{(2)}\left(z_{1}, z_{2}, r\right),
\end{aligned}
$$

and

$$
\delta=-\frac{1}{8 \sigma_{0}} \int \mathrm{d} z_{1} \int \mathrm{d} \mathbf{r}_{12} u^{\prime}(r) r\left(1-3 s^{2}\right) \rho_{1}^{(2)}\left(z_{1}, z_{2}, r\right) .
$$

In these equations, $u(r)$ is the interaction potential, $\rho_{0}^{(2)}\left(z_{1}, z_{2}, r\right)$ is the pair density of the planar interface, $\rho_{1}^{(2)}\left(z_{1}, z_{2}, r\right)$ is the first order correction to an expansion of the pair density in powers of the curvature, $r=r_{12} \equiv\left|\mathbf{r}_{12}\right|, s$ $=\cos \theta_{12}$, and $z_{2}=z_{1}+s r$, and it is understood that the integration over $z$ runs from the liquid to the vapor phase and where the $z=0$ plane is chosen to coincide with the equimolar surface. The determination of the Tolman length in molecular dynamics (MD) simulations via Eq. (6) is inconsistent with the results of DFT. In order to investigate this discrepancy, it seems prudent to return to Eq. (4) and to determine $\Delta p$ directly from computer simulation. Advances in computing power now make this approach feasible.

There are two methods of determining $\Delta p$. One can simply ignore the interfacial regions and evaluate the bulk liquid and vapor pressures via the pressure tensor. To get better insight into the numerical accuracy of this method, we also derive a second route to $\Delta p$, which uses an integral over the components of the pressure tensor throughout the interfacial region. In Sec. II, we discuss the pressure tensor and derive several expressions for $\Delta p$ via the second route. We evaluate $\Delta p$ from both methods using MD simulations of liquid drops in equilibrium with their vapor. The details of these simulations are given in Sec. III, and we discuss their results in Sec. IV. Finally, in Sec. V we present some conclusions.

\section{THE LOCAL PRESSURE TENSOR}

In this section, we derive an alternative route to $\Delta p$ using the components of the pressure tensor. For two phases in contact, the condition for mechanical equilibrium is

$$
\boldsymbol{\nabla} \cdot \mathbf{p}(\mathbf{r})=0,
$$

where $\mathbf{p}(\mathbf{r})$ is the position-dependent pressure tensor. For liquid drops surrounded by vapor, the system is spherically symmetric and the pressure tensor has only two components. It can be written as

$$
\mathbf{p}(\mathbf{r})=p_{\mathrm{N}}(r) \hat{\mathbf{e}}_{r} \hat{\mathbf{e}}_{r}+p_{\mathrm{T}}(r)\left(\mathbf{1}-\hat{\mathbf{e}}_{r} \hat{\mathbf{e}}_{r}\right)
$$

where $p_{\mathrm{N}}(r)$ and $p_{\mathrm{T}}(r)$ are the components normal to and tangential to the interface, respectively, and depend on the distance $r$ from the center of the drop. Note that both $p_{\mathrm{N}}(r)$ and $p_{\mathrm{T}}(r)$ become equal to the homogeneous or bulk pressure deep within the liquid or vapor phases. The unit vector is defined by $\hat{\mathbf{e}}_{r}=\mathbf{r} /|\mathbf{r}|$. Inserting these into the definition for mechanical equilibrium, Eq. (8) gives

$$
\frac{\partial p_{\mathrm{N}}(r)}{\partial r}=-\frac{2}{r}\left[p_{\mathrm{N}}(r)-p_{\mathrm{T}}(r)\right]
$$

Integration of this expression from deep inside the droplet to deep into the vapor phase gives

$$
\Delta p=2 \int_{0}^{\infty} \mathrm{d} r \frac{1}{r}\left[p_{\mathrm{N}}(r)-p_{\mathrm{T}}(r)\right] .
$$

The normal and tangential components of the pressure tensor have contributions from the kinetic motion of the particles and from the interaction between particles. For the normal component, this is

$$
p_{\mathrm{N}}(r)=\rho(r) k_{\mathrm{B}} T+p_{\mathrm{N}}^{\mathrm{C}}(r),
$$

and similarly for $p_{\mathrm{T}}(r)$. The term $\rho(r) k_{\mathrm{B}} T$ accounts for the ideal gas contribution. Since the second term is due to the interactions between pairs of molecules, some arbitrariness is involved in locating its contribution to the local pressure tensor. Different choices can be made which can be traced back to defining a certain contour connecting the pair of particles. When this contour is taken to be a straight line, the corresponding expression for the pressure tensor is that defined by Irving and Kirkwood. ${ }^{17}$ The normal component of the Irving-Kirkwood pressure tensor will be determined for each droplet in our simulations. The details of the calculation can be found in Thompson. ${ }^{13}$ It should be emphasized that this route is identical to the virial route when the IrvingKirkwood definition for the local pressure tensor is used. ${ }^{18}$

The difference between the normal and tangential components of the pressure tensor $p_{\mathrm{N}}(r)-p_{\mathrm{T}}(r)$ can also be determined directly from the Irving-Kirkwood expression for the pressure tensor, which involves the pair density of the spherical interface $\rho_{\mathrm{s}}^{(2)}\left(\mathbf{r}_{1}, \mathbf{r}_{2}\right),{ }^{17,18}$

$$
\begin{aligned}
p_{\mathrm{N}}\left(r_{1}\right)-p_{\mathrm{T}}\left(r_{1}\right)= & \frac{1}{4} \int \mathrm{d} \mathbf{r}_{12} \int_{0}^{1} \mathrm{~d} \alpha \frac{u^{\prime}\left(r_{12}\right)}{r_{12}} \rho_{\mathrm{s}}^{(2)}\left(\mathbf{r}_{1}-\alpha \mathbf{r}_{12},\right. \\
& \left.\mathbf{r}_{1}+(1-\alpha) \mathbf{r}_{12}\right)\left[\mathbf{r}_{12}-3\left(\mathbf{r}_{12} \cdot \hat{\mathbf{e}}_{r_{1}}\right)^{2}\right]
\end{aligned}
$$

where $\alpha$ is a dimensionless integration variable. Inserting this into Eq. (11) gives

$$
\begin{gathered}
\Delta p=\frac{1}{2} \int_{0}^{\infty} \mathrm{d} r_{1} \frac{1}{r_{1}} \int \mathrm{d} \mathbf{r}_{12} \int_{0}^{1} \mathrm{~d} \alpha \frac{u^{\prime}\left(r_{12}\right)}{r_{12}} \rho_{\mathrm{s}}^{(2)}\left(\mathbf{r}_{1}-\alpha \mathbf{r}_{12},\right. \\
\left.\mathbf{r}_{1}+(1-\alpha) \mathbf{r}_{12}\right) \frac{1}{r_{12}^{2}}\left[\mathbf{r}_{12}^{2} \cdot \mathbf{r}_{1}^{2}-3\left(\mathbf{r}_{12} \cdot \mathbf{r}_{1}\right)^{2}\right]
\end{gathered}
$$

The integration variable for the first integral can be changed 
from $\mathrm{d} r_{1}$ to $\mathrm{d} \mathbf{r}_{1}$. In doing so, we gain both a factor of $1 / 4 \pi$ and a factor of $1 / r_{1}^{2}$,

$$
\begin{aligned}
\Delta p= & \frac{1}{8 \pi} \int \mathrm{d} \mathbf{r}_{1} \int \mathrm{d} \mathbf{r}_{12} \int_{0}^{1} \mathrm{~d} \alpha \frac{u^{\prime}\left(r_{12}\right)}{r_{12}} \frac{1}{r_{1}^{5}}\left[r_{12}^{2} r_{1}^{2}\right. \\
& \left.-3\left(\mathbf{r}_{12} \cdot \mathbf{r}_{1}\right)^{2}\right] \rho_{\mathrm{s}}^{(2)}\left(\mathbf{r}_{1}-\alpha \mathbf{r}_{12}, \mathbf{r}_{1}+(1-\alpha) \mathbf{r}_{12}\right) .
\end{aligned}
$$

In order to proceed, we note that if we define $\mathbf{R}_{1}=\mathbf{r}_{1}-\alpha \mathbf{r}_{12}$, then

$$
\begin{aligned}
\frac{\partial}{\partial \alpha}\left[\frac{\mathbf{r}_{1} \cdot \mathbf{r}_{12}}{\mathbf{r}_{1}^{5}}\right] & =\frac{\partial}{\partial \alpha}\left[\frac{\mathbf{R}_{1} \cdot \mathbf{r}_{12}+\alpha \mathbf{r}_{12}^{2}}{\left(R_{1}^{2}+2 \alpha \mathbf{R}_{1} \cdot \mathbf{r}_{12}+\alpha^{2} r_{12}^{2}\right)^{3 / 2}}\right] \\
& =\frac{1}{r_{1}^{5}}\left[r_{12}^{2} r_{1}^{2}-3\left(\mathbf{r}_{12} \cdot \mathbf{r}_{1}\right)^{2}\right]
\end{aligned}
$$

which appears in the integrand of Eq. (15). After redefining our integration variable $\mathbf{r}_{1} \rightarrow \mathbf{r}_{1}+\alpha \mathbf{r}_{12}$, Eq. (15) becomes

$$
\begin{aligned}
\Delta p= & \frac{1}{8 \pi} \int \mathrm{d} \mathbf{r}_{1} \int \mathrm{d} \mathbf{r}_{12} \frac{u^{\prime}\left(r_{12}\right)}{r_{12}} \\
& \times \rho_{\mathrm{s}}^{(2)}\left(\mathbf{r}_{1}, \mathbf{r}_{2}\right) \int_{0}^{1} \mathrm{~d} \alpha \frac{\partial}{\partial \alpha}\left[\frac{\mathbf{r}_{1} \cdot \mathbf{r}_{12}+\alpha r_{12}^{2}}{\left|\mathbf{r}_{1}+\alpha \mathbf{r}_{12}\right|^{2}}\right] .
\end{aligned}
$$

Upon integration over $\alpha$, the expression for $\Delta p$ becomes

$$
\begin{aligned}
\Delta p= & \frac{1}{8 \pi} \int \mathrm{d} \mathbf{r}_{1} \int \mathrm{d} \mathbf{r}_{12} \frac{u^{\prime}\left(r_{12}\right)}{r_{12}}\left[\frac{\mathbf{r}_{2} \cdot\left(\mathbf{r}_{2}-\mathbf{r}_{1}\right)}{r_{2}^{3}}\right. \\
& \left.-\frac{\mathbf{r}_{1} \cdot\left(\mathbf{r}_{2}-\mathbf{r}_{1}\right)}{r_{1}^{3}}\right] \rho_{\mathrm{s}}^{(2)}\left(\mathbf{r}_{1}, \mathbf{r}_{2}\right) .
\end{aligned}
$$

From here, two courses of action are available. One can make use of the symmetry between particles 1 and 2 and simplify Eq. (18) to

$$
\Delta p=\frac{-1}{4 \pi} \int \mathrm{d} \mathbf{r}_{1} \int \mathrm{d} \mathbf{r}_{2} \frac{u^{\prime}\left(r_{12}\right)}{r_{12}}\left[\frac{\mathbf{r}_{1} \cdot \mathbf{r}_{12}}{r_{1}^{3}}\right] \rho_{\mathrm{s}}^{(2)}\left(\mathbf{r}_{1}, \mathbf{r}_{2}\right)
$$

Choosing $\mathbf{r}_{1}=r_{1} \hat{\mathbf{z}}$,

$$
\Delta p=\frac{-1}{4 \pi} \int \mathrm{d} \mathbf{r}_{1} \int \mathrm{d} \mathbf{r}_{12} u^{\prime}\left(r_{12}\right) s \rho_{\mathrm{s}}^{(2)}\left(r_{1}, r_{2}, r_{12}\right) .
$$

Not using the 1-2 symmetry but choosing $\mathbf{r}_{1}=r_{1} \hat{\mathbf{z}}$ in Eq. (18) immediately gives

$$
\begin{aligned}
\Delta p= & \frac{1}{2} \int_{0}^{\infty} \mathrm{d} r_{1} \int \mathrm{d} \mathbf{r}_{12} \frac{u^{\prime}\left(r_{12}\right)}{r_{12}}\left[\frac{\left(r_{1}^{3}-r_{2}^{3}\right) s r_{12}+r_{1}^{2} r_{12}^{2}}{r_{2}^{3}}\right] \\
& \times \rho_{\mathrm{s}}^{(2)}\left(\mathbf{r}_{1}, \mathbf{r}_{2}\right) .
\end{aligned}
$$

This is the equation which is used to determine $\Delta p$ next to the determination of the individual bulk pressures. The limits on the integration are from deep in the liquid phase to deep in the vapor phase. In practice, the lower limit to this integral was never taken to be less than $2.5 \sigma$ due to the unavoidably poor statistics related to the small volume of the spherical shell used in the averaging. This results in a larger uncertainty for the smallest droplets.
When Eq. (21) is expanded to second order in $1 / R_{\mathrm{e}}$ and compared to Eq. (4), one retrieves for $\sigma_{0}$ the virial expression in Eq. (5). For the Tolman length, one finds that it is the sum of two contributions

$$
\begin{aligned}
\delta= & \frac{1}{8 \sigma_{0}} \int_{-\infty}^{\infty} \mathrm{d} z_{1} \int \mathrm{d} \mathbf{r}_{12} u^{\prime}\left(r_{12}\right) r_{12}\left(1-3 s^{2}\right)\left(z_{1}+z_{2}\right) \\
& \times \rho_{0}^{(2)}\left(z_{1}, z_{2}, r_{12}\right)-\frac{1}{4 \sigma_{0}} \int_{-\infty}^{\infty} \mathrm{d} z_{1} \int \mathrm{d} \mathbf{r}_{12} u^{\prime}\left(r_{12}\right) r_{12} \\
& \times\left(1-3 s^{2}\right) \rho_{1}^{(2)}\left(z_{1}, z_{2}, r_{12}\right) .
\end{aligned}
$$

This expression for $\delta$ is therefore consistent with the virial expressions for $\delta$ in Eqs. (6) and (7), which is a consequence of the more general observation that the local pressure tensor route is always consistent with the virial route as long as the Irving-Kirkwood definition for the pressure tensor is used. ${ }^{18}$ However, as mentioned above, the determination from simulations of $\Delta p$ through Eq. (21), which results in the Tolman length being determined by Eq. (22), gives a value for the Tolman length which differs from that determined from Eq. (6) or (7), separately.

\section{SIMULATION DETAILS}

In order to extract the Tolman length from the dependence of $\Delta p$ on the droplet radius, it was necessary to perform simulations of variously sized droplets as well as of planar interfaces of varying surface area. All simulations were performed at a reduced temperature of $T^{*} \equiv k_{\mathrm{B}} T / \varepsilon$ $=0.9$.

The simulations consisted of MD simulations of particles interacting through the Lennard-Jones potential

$$
u_{\mathrm{LJ}}(r)=4 \varepsilon\left[\left(\frac{\sigma}{r}\right)^{12}-\left(\frac{\sigma}{r}\right)^{6}\right]
$$

where $\varepsilon$ is the depth of the potential well and $\sigma$ is the molecular diameter (not to be confused with the surface tension). The potential is cut-off at a distance of $r_{\mathrm{c}}=2.5 \sigma$. In addition, the potential is shifted such that it is zero at the cutoff. Thus, the actual potential used in the simulations is

$$
u(r)=\left\{\begin{array}{ll}
u_{\mathrm{LJ}}(r)-u_{\mathrm{LJ}}\left(r_{\mathrm{c}}\right) & r<r_{\mathrm{c}} \\
0 & r \geq r_{\mathrm{c}}
\end{array} .\right.
$$

As usual for MD simulations, all quantities are reduced by the appropriate factors of $\varepsilon, \sigma$, Boltzmann constant $k_{\mathrm{B}}$, and the particle mass $m$. In general, due to the finite cutoff, the physical quantities obtained in the simulations are only approximations of those in real systems. It is known, for example, that the value of the surface tension changes substantially when the cutoff is increased from 2.5 to $7.33 \sigma{ }^{19}$

The simulations used both the linklist and the pairlist method. The lists were updated every seven timesteps. The reduced timestep was $\Delta t=0.01$. The temperature was kept constant by using a Nosé-Hoover chain for each particle. ${ }^{20}$ Each chain had two masses, each of mass 10. It was found that due to the extremely small nature of the difference between the normal component of the bulk pressure inside and outside the droplet, inhomogeneities in the temperature pro- 
TABLE I. Values for the number of particles, number of timesteps, box size, liquid and vapor densities, pressure, surface tension, Tolman length, and width of the interface for the planar simulations.

\begin{tabular}{cccccccc}
\hline \hline$N$ & Timesteps & $L_{x} \times L_{y} \times L_{z}$ & $\rho_{\ell}$ & $\rho_{v}$ & $p$ & $\sigma_{0}$ & $\delta$ \\
\hline 10000 & $3.0 \times 10^{8}$ & $15 \times 15 \times 150$ & 0.664682 & 0.044999 & 0.031401 & 0.232182 & 0.081 \\
11000 & $3.0 \times 10^{8}$ & $20 \times 20 \times 100$ & 0.664688 & 0.044975 & 0.031401 & 0.229766 & 0.183 \\
22000 & $3.0 \times 10^{8}$ & $25 \times 25 \times 100$ & 0.664712 & 0.044990 & 0.031386 & 0.228722 & 0.167 \\
26000 & $3.0 \times 10^{8}$ & $30 \times 30 \times 100$ & 0.664716 & 0.044973 & 0.031391 & 0.228418 & 0.196 \\
100000 & $1.8 \times 10^{8}$ & $50 \times 50 \times 150$ & 0.664735 & 0.044973 & 0.031386 & 0.228014 & 0.208 \\
& & $\infty \times \infty \times \infty$ & 0.664743 & 0.044974 & 0.031382 & 0.227774 & 0.207 \\
\hline \hline
\end{tabular}

file, due to more computationally inexpensive thermostats, had a measurable effect on the value of $\Delta p$ via Eq. (12). By using a Nosé-Hoover chain for each particle in the simulation, the fluctuations in the temperature profile were kept to a minimum; typically, the average temperature was within $0.0002 \%$ of $T^{*}=0.90$ with maximum fluctuations of less than $0.02 \%$.

The total momentum of the system was set to zero every 100 timesteps to correct for drift due to round-off errors in the calculation; this is more important for smaller systems than for larger systems. At the same time, the location of every particle was shifted so that the center of mass of the drop-defined using location of all the particles within a radius of $R_{\mathrm{e}}+2$ from the origin - was in the center of the box in order to prevent drift in the location of the droplet. The statistical error in various quantities was determined by averaging over subintervals of 100000 timesteps. The details for the simulations for the planar interfaces and for each droplet size are given in Tables I and II, respectively.

The simulations took place in a rectangular box with periodic boundary conditions on all sides. The shape of the box determined the geometry of the equilibrated liquid phase as discussed in previous work. ${ }^{9}$ The initial configurations for the simulations contained both phases, a high-density slab or sphere surrounded by a low-density "vapor," with the particles on a fcc lattice. The lattice spacing was appropriate for a liquid or for a vapor density, depending on the phase. The desired geometry of the equilibrated phase was already present in the initial configuration. For the simulations with droplets, the approximate number of particles necessary for a droplet of radius $R_{\mathrm{e}}$ was determined via the relation ${ }^{12}$

$$
N=\rho_{\ell} \frac{4 \pi}{3} R_{\mathrm{e}}^{3}+\rho_{v}\left(L^{3}-\frac{4 \pi}{3} R_{\mathrm{e}}^{3}\right),
$$

where $L$ is the length of a side of the square computational box and $\rho_{\ell}$ and $\rho_{v}$ are the bulk liquid and vapor densities. Note that this relation was only used in constructing an initial configuration. The equimolar radius used in the analysis was determined via a different expression [see Eq. (27)]. All configurations were allowed to fully equilibrate for at least $1 \times 10^{7}$ timesteps, the equivalent of $10 \mathrm{~ns}$.

\section{RESULTS AND ANALYSIS}

The results from the planar simulations and the droplet simulations will be discussed separately, beginning with those from the planar systems. An extensive exploration of the size-dependence of the planar system was carried out, motivated by an apparent inconsistency in the values of the surface tension of a planar interface and the extrapolated infinite-radius limit from the droplet simulations. Such finitesize effects were recently reported by Biscay et $a l .{ }^{21}$ Five different planar systems were simulated, the details of which are given in Table I. All five simulations were carried out in a box that resulted in a square slab of liquid as the equilib-

TABLE II. Values for the number of particles, number of timesteps, box size, equimolar radius, liquid and vapor densities, and two different determinations of $\Delta p$ for the droplet simulations.

\begin{tabular}{cccccccc}
\hline \hline$N$ & Timesteps & $L$ & $R_{\mathrm{e}}$ & $\rho_{\ell}$ & $\rho_{v}$ & $\Delta p$ & $\Delta p^{\text {bulk }}$ \\
\hline 8642 & $2.0 \times 10^{8}$ & 50 & 9.033 & 0.678697 & 0.054028 & 0.049890 & 0.051377 \\
9295 & $2.0 \times 10^{8}$ & 50 & 10.128 & 0.677278 & 0.052803 & 0.045172 & 0.045762 \\
10420 & $2.0 \times 10^{8}$ & 50 & 11.489 & 0.675900 & 0.051737 & 0.039886 & 0.040133 \\
17000 & $2.0 \times 10^{8}$ & 60 & 13.240 & 0.674525 & 0.050692 & 0.034736 & 0.034839 \\
20000 & $1.0 \times 10^{8}$ & 60 & 15.240 & 0.673292 & 0.049836 & 0.030067 & 0.030183 \\
30000 & $1.0 \times 10^{8}$ & 70 & 17.125 & 0.672405 & 0.049279 & 0.026732 & 0.026776 \\
43000 & $1.0 \times 10^{8}$ & 80 & 19.050 & 0.671642 & 0.048773 & 0.024022 & 0.024041 \\
50000 & $1.0 \times 10^{8}$ & 80 & 21.315 & 0.670954 & 0.048338 & 0.021457 & 0.021475 \\
80000 & $1.0 \times 10^{8}$ & 100 & 23.049 & 0.670516 & 0.048080 & 0.019837 & 0.019855 \\
90000 & $1.5 \times 10^{8}$ & 100 & 25.304 & 0.670011 & 0.047783 & 0.018061 & 0.018066 \\
134000 & $1.5 \times 10^{8}$ & 120 & 27.089 & 0.669672 & 0.047575 & 0.016870 & 0.016880 \\
150000 & $1.0 \times 10^{8}$ & 120 & 29.694 & 0.669257 & 0.047343 & 0.015391 & 0.015387 \\
246000 & $0.7 \times 10^{8}$ & 150 & 32.197 & 0.668898 & 0.047135 & 0.014190 & 0.014192 \\
270000 & $0.7 \times 10^{8}$ & 150 & 34.983 & 0.668595 & 0.046969 & 0.013050 & 0.013052 \\
579000 & $0.5 \times 10^{8}$ & 180 & 49.132 & 0.667495 & 0.046370 & 0.009281 & 0.009285 \\
\hline \hline
\end{tabular}




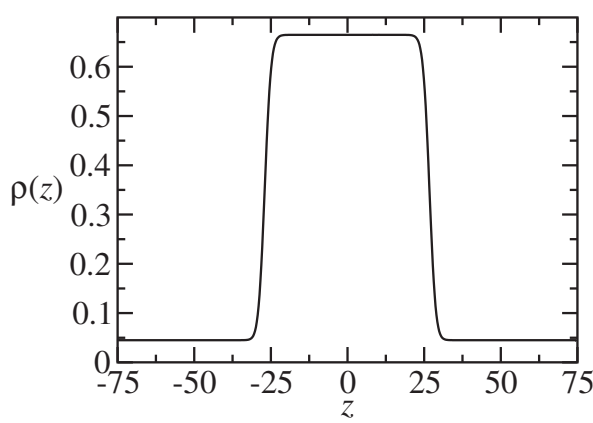

FIG. 1. Density profile for a planar liquid slab for the system with $N$ $=100000$.

rium configuration. For the simulations of drops, the simulation box was large enough that properties of the droplet were independent of the box size.

Figure 1 shows the density profile for the largest system studied. A measure of the width of the interface can be obtained by fitting the density profiles to a hyberbolic tangent ${ }^{13}$

$$
\rho(z)=\frac{1}{2}\left(\rho_{\ell}+\rho_{v}\right)-\frac{1}{2}\left(\rho_{\ell}-\rho_{v}\right) \tanh \left(\frac{2}{D}\left(z-z_{\mathrm{e}}\right)\right) .
$$

Fitting the density profile this way does not accurately capture the details of the profile in the interfacial region, so the width $D$ is only intended to be an approximate measure of the width of the interface. The width of the profile increases slightly as the surface area increases. The pressure profiles for both the normal and tangential components of the pressure tensor are shown for the same system in Fig. 2.

As seen in Table I, the bulk liquid density, width of the interface, the surface tension, and, to a lesser extent, the normal component of the pressure tensor, depend very weakly on the surface area. The bulk vapor density had no obvious dependence on the surface area. The surface tension and bulk pressure decreased as the surface area increased, while the bulk liquid density and the approximate width of the interface were observed to increase with increasing surface area. The magnitude of the change was, in all cases, slight. For example, the difference between the largest and smallest values of the pressure was just $0.05 \%$, while that for the bulk liquid densities was $0.008 \%$. Nevertheless, the values for these quantities used for comparison with the results from the droplet simulations are those given by a linear extrapolation (as a function of the inverse area) to the limit of infi-

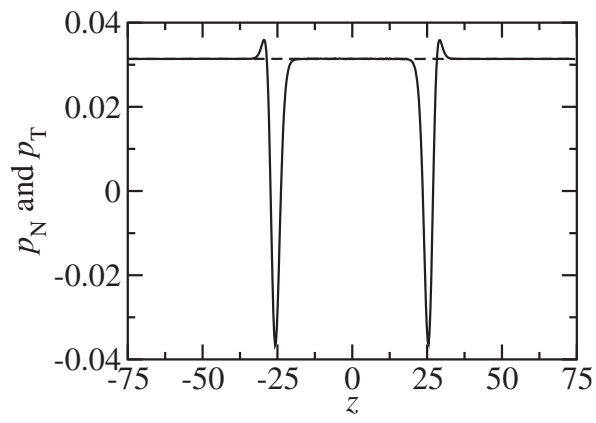

FIG. 2. The components of the pressure tensor for the same system as in Fig. 1. The dashed line is the normal component and the solid line is the tangential component.

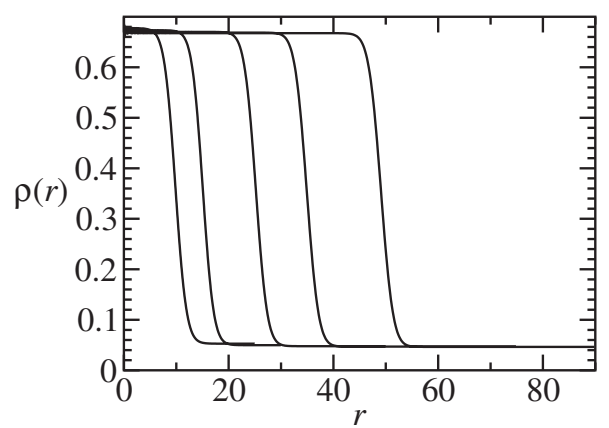

FIG. 3. Density profiles for five different system sizes. In order of increasing $R_{\mathrm{e}}$, they are $N=9295, N=20000, N=90000, N=270000$, and $N$ $=579000$. Details for each system can by found in Table II.

nite box size. The reason for the investigation of the areadependence of the properties of the planar system was the disagreement of planar surface tension for the $N=11000$ system (which was used in previous work) ${ }^{9}$ and the extrapolation to large droplet sizes for Eq. (4). The discrepancy is due to the cutoff of long wavelength capillary waves in the planar simulations. The larger box sizes allow for a larger spectrum of capillary waves and hence a slight sizedependence to the surface tension.

The Tolman length was also calculated for each system size using Eq. (6) and is given in Table I. It, too, shows a dependence on system size, increasing with increasing surface area. As is clear from Table I, $\delta$ is sensitive to the number of particles, but a full exploration of this dependence is not the goal here since it is unlikely to resolve the sign discrepancy discussed in Sec. I. The value of $\delta$ extrapolated to infinite surface area is $\delta=+0.207 \pm 0.002$. While this is in general agreement with values found previously, ${ }^{9,10}$ it is not in agreement with results from DFT nor with the value determined by the curvature dependence of $\Delta p$ as calculated using Eq. (21), as will be shown below.

The density profile for a number of representative droplets is shown in Fig. 3. These include droplets with equimolar radii of $R_{\mathrm{e}}=10,15,25,35$, and 49 . The equimolar radius is determined by setting the excess number density to zero

$$
\Gamma=\int \mathrm{d} r r^{2}\left[\rho(r)-\rho_{\mathrm{b}}\right]=0,
$$

where $\rho_{\mathrm{b}}=\rho_{\ell}$ for $r<R_{\mathrm{e}}$ and $\rho_{\mathrm{b}}=\rho_{v}$ for $r>R_{\mathrm{e}}$. As the radius of the droplet increases, the density of both the liquid and vapor phases decreases. Figure 4 shows the profile of the

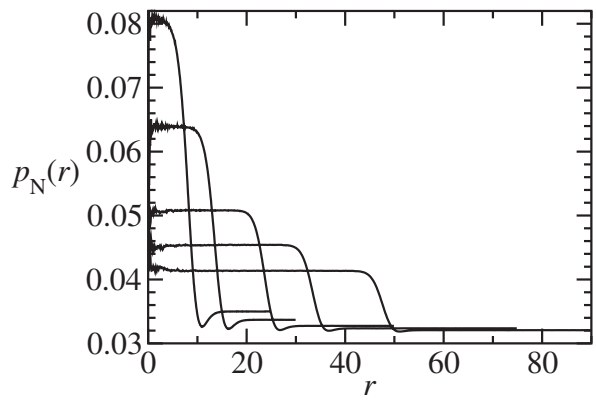

FIG. 4. Normal component of the pressure tensor for the five systems in Fig. 3. Details for each system can by found in Table II. 


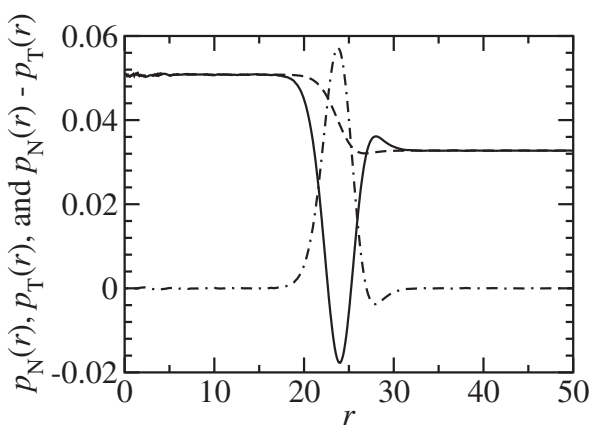

FIG. 5. Normal and tangential components of the pressure tensor for the system of $N=90000$. The dashed line is the normal component, the solid line is the tangential component, and the dotted-dashed line is their difference, $p_{\mathrm{N}}-p_{\mathrm{T}}$.

normal component of the pressure tensor as a function of $r$ for the same radii as in Fig. 3. As the equimolar radius increases, both the bulk liquid and bulk vapor pressures decrease. A minimum in each pressure profile is seen at a radius slightly larger than the equimolar radius. This is consistent with what has been seen in other simulations, ${ }^{22}$ though unlike that work, this minimum was never observed to be less than zero. The magnitude of this minimum decreases with increasing $R_{\mathrm{e}}$. Also in contrast with Ref. 22, the $p_{\mathrm{N}}(r)$ profile increases monotonically from the minimum with increasing $r$ and no maximum was observed on the vapor side of the interface. Figure 5 shows the behavior of the normal and tangential components of the pressure tensor for a representative droplet. Also shown is the difference $p_{\mathrm{N}}-p_{\mathrm{T}}$ that appears in the integrand of Eq. (11). As can be clearly seen, the value of this integrand is only nonzero in the interfacial region.

Figures 6 and 7 show the dependence of the bulk density of the liquid and vapor phases, respectively, on the curvature. The value for each at $1 / R_{\mathrm{e}}=0$ was taken from the infinite limit of the planar simulations discussed above. In both cases, the value from the planar simulations is in complete agreement with the infinite-radius limit. Figure 8 shows a similar plot for value of the normal component of the pressure tensor in the liquid and vapor phases as well as that from the planar simulations. The error bars for this plot are smaller than the symbols. Here, too, the extrapolation of the droplet data to the infinite $R$ limit is in complete agreement with the results from the planar simulations.

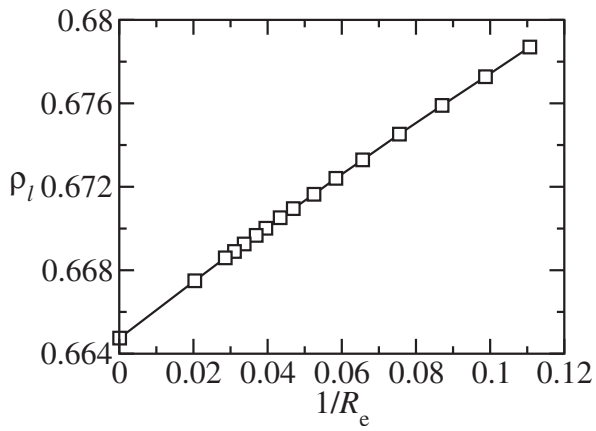

FIG. 6. Dependence of the bulk liquid density on the curvature. The error bars are smaller than the symbols. The data point at $1 / R_{\mathrm{e}}=0$ is an extrapolation to the infinite area limit from the planar simulations.

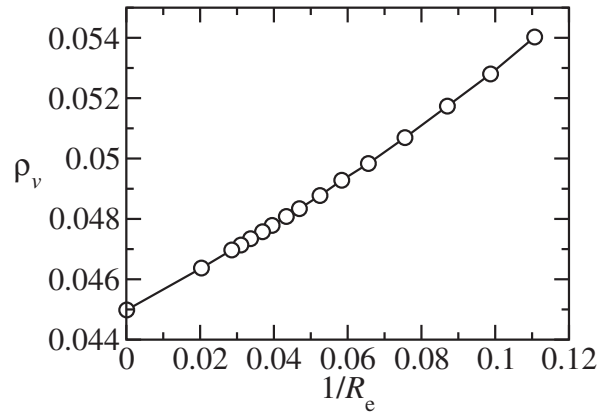

FIG. 7. Dependence of the bulk vapor density on the curvature. The error bars are smaller than the symbols. The data point at $1 / R_{\mathrm{e}}=0$ is an extrapolation to the infinite area limit from the planar simulations.

Finally, Fig. 9 shows a plot of $\Delta p R_{\mathrm{e}} / 2$ versus $1 / R_{\mathrm{e}}$. The two sets of data in Fig. 9 are those using the $\Delta p$ calculated using Eq. (21), labeled $\Delta p$, and those determined from the bulk values of the normal component of the IrvingKirkwood pressure tensor, labeled $\Delta p^{\text {bulk }}$, as given in Table II. The error bars in this figure were obtained from the standard deviations for the average values of both $\Delta p$ and $R_{\mathrm{e}}$ for each subinterval of 100000 timesteps. The large error for the smallest droplets is due to the difficulty of determining the bulk pressure of such a small liquid phase (see Fig. 4). Rearranging Eq. (4),

$$
\frac{1}{2} \Delta p R_{\mathrm{e}}=\sigma_{0}-\sigma_{0} \delta \frac{1}{R_{\mathrm{e}}}+\ldots
$$

In the limit of large $R_{\mathrm{e}}$, the higher order terms may be neglected. In this limit, which we take to be $R_{\mathrm{e}}>16$, the slope of the best fit line to the data in Fig. 9 is $-\sigma_{0} \delta$, while the intercept is $\sigma_{0}$. As is clear from the figure, there is a positive slope to the linear fit, which corresponds to a negative Tolman length. Two fits were performed to this data, with the results given in Table III. One fit used a fixed intercept equal to the surface tension of an infinite planar interface, labeled $\sigma_{0}$ in Table III, and another where the intercept was itself a fit parameter, labeled $\sigma_{0}$ (fit). The lines in Fig. 9 are the fits using the fixed value of $\sigma_{0}$. All four fits are in good agreement with each other. The Tolman length as determined from a direct calculation of the bulk pressures is $\delta=-0.10 \pm 0.02$. This is in excellent agreement with predictions from DFT. ${ }^{8}$ This is in contrast with the Tolman length as calculated via

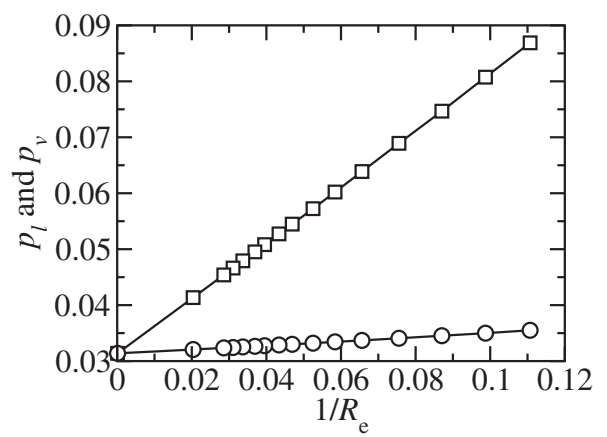

FIG. 8. Dependence of the bulk pressures on the curvature. The squares are the bulk liquid pressures and the circles are the bulk vapor pressures. The error bars are smaller than the symbols. The data point at $1 / R_{\mathrm{e}}=0$ is an extrapolation to the infinite area limit from the planar simulations. 


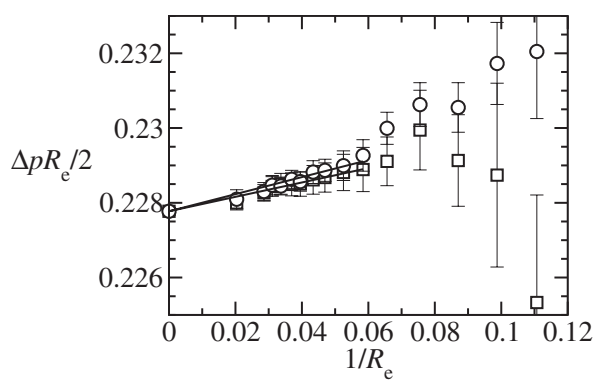

FIG. 9. Dependence of $\Delta p R_{\mathrm{e}} / 2$ on the curvature. The squares are from Eq (21) and the circles use $\Delta p^{\text {bulk }}=p_{\ell}-p_{v}$, the difference in the bulk pressures as determined by the Irving-Kirkwood pressure tensor. The fits are using the fixed value of $\sigma_{0}$ in Table III. The data point at $1 / R_{\mathrm{e}}=0$ is an extrapolation to the infinite area limit for the surface tension of a planar interface.

Eq. (6) in this work, as well as previously. ${ }^{9}$ The reason for this discrepancy is not yet clear.

In order to further investigate this inconsistency, we have revisited the determination of the Tolman length from DFT in more detail. The details of this are given in the Appendix, and a comparison of the DFT results with those from the simulations is given in Table IV. As with the virial route, two expressions for the Tolman length are found via DFT,

$$
\begin{aligned}
& \delta=\frac{1}{4 \sigma_{0}} \int_{-\infty}^{\infty} \mathrm{d} z_{1} \int \mathrm{d} \mathbf{r}_{12} u_{\mathrm{att}}(r) r^{2}\left(1-s^{2}\right) z_{1} \rho_{0}^{\prime}\left(z_{1}\right) \rho_{0}^{\prime}\left(z_{2}\right), \\
& \delta=\frac{1}{4 \sigma_{0}} \int_{-\infty}^{\infty} \mathrm{d} z_{1} \int \mathrm{d} \mathbf{r}_{12} u_{\mathrm{att}}(r) r^{2}\left(1-s^{2}\right) \rho_{0}^{\prime}\left(z_{1}\right) \rho_{1}^{\prime}\left(z_{2}\right) .
\end{aligned}
$$

One can show that there is a high degree of consistency between these DFT expressions for $\delta$ and the virial expressions in Eqs. (6) and (7) when the pair density is approximated by the product of the densities in the virial expressions, e.g., $\rho_{0}^{(2)}\left(z_{1}, z_{2}, r\right) \approx \rho_{0}\left(z_{1}\right) \rho_{0}\left(z_{2}\right)$. The resulting expressions

$$
\begin{aligned}
\delta= & -\frac{1}{8 \sigma_{0}} \int_{-\infty}^{\infty} \mathrm{d} z_{1} \int \mathrm{d} \mathbf{r}_{12} u_{\text {att }}^{\prime}(r) r\left(1-3 s^{2}\right) \\
& \times\left(z_{1}+z_{2}\right) \rho_{0}\left(z_{1}\right) \rho_{0}\left(z_{2}\right), \\
\delta= & -\frac{1}{8 \sigma_{0}} \int_{-\infty}^{\infty} \mathrm{d} z_{1} \int \mathrm{d} \mathbf{r}_{12} u_{\text {att }}^{\prime}(r) r\left(1-3 s^{2}\right)\left[\rho_{0}\left(z_{1}\right) \rho_{1}\left(z_{2}\right)\right. \\
& \left.+\rho_{1}\left(z_{1}\right) \rho_{0}\left(z_{2}\right)\right],
\end{aligned}
$$

reduce to Eqs. (29) and (30) via integration by parts. This is explicitly shown in the appendix of Ref. 23 . We have verified that all four expressions in Eqs. (29)-(32) give the same value for $\delta$ in DFT (see Tables IV and V). The value ob-

TABLE III. Fit details for the evaluation of the Tolman length (see text for details).

\begin{tabular}{lcccc}
\hline \hline & $\sigma_{0}$ & $\delta$ & $\sigma_{0}($ fit $)$ & $\delta$ \\
\hline$\Delta p$ & 0.227774 & -0.084522 & 0.227730 & -0.089158 \\
$\Delta p^{\text {bulk }}$ & 0.227774 & -0.100244 & 0.227650 & -0.113204 \\
\hline \hline
\end{tabular}

TABLE IV. Listed are numerical values for the various quantities obtained in the simulations and DFT: reduced temperature, reduced critical temperature, liquid and vapor densities, pressure, surface tension, and the Tolman length evaluated in two ways [in the simulations, $\delta$ is evaluated from the direct evaluation of $\Delta p$ and $\delta_{\text {planar }}$ from Eq. (6). In DFT, $\delta=\delta_{\text {planar }}$, which is evaluated from any of Eqs. (29)-(32)]. MD values are for the extrapolation to the infinite area limit.

\begin{tabular}{lcc}
\hline \hline & MD & DFT \\
\hline$T^{*}$ & 0.90 & 0.911297 \\
$T_{\mathrm{c}}^{*}$ & 1.08 & 1.230286 \\
$\rho_{\ell}$ & 0.664743 & 0.645403 \\
$\rho_{v}$ & 0.044974 & 0.025634 \\
$p$ & 0.031382 & 0.019920 \\
$\sigma_{0}$ & 0.227774 & 0.33720 \\
$\delta$ & -0.10 & -0.1462 \\
$\delta_{\text {planar }}$ & 0.207 & -0.1462 \\
\hline \hline
\end{tabular}

tained in this way, $\delta=-0.1426$, seems to be consistent with the value obtained in the simulations via the difference in pressure $\Delta p$ for liquid droplets. To obtain this number, we have matched the DFT to the simulations rather straightforwardly: taking for $u_{\text {att }}(r)$ the attractive part of the LennardJones potential and setting the hard sphere diameter simply equal to $\sigma$. One might therefore expect to obtain even better agreement with the DFT results when a more sophisticated matching is made. The important conclusion is, however, that the virial expressions in Eqs. (6) and (7) used in the MD simulations seem to give inconsistent results, the analogous DFT expressions in Eqs. (31) and (32) are consistent.

Finally, Eq. (6) can be split into two parts

$$
\delta=\delta_{1}+\delta_{2},
$$

where $\delta_{1}$ and $\delta_{2}$ are defined by

$$
\delta_{1}=-\frac{1}{8 \sigma_{0}} \int \mathrm{d} z_{1} \int \mathrm{d} \mathbf{r}_{12} u^{\prime}(r) r\left(1-3 s^{2}\right)\left(2 z_{1}\right) \rho_{0}^{(2)}\left(z_{1}, z_{2}, r\right),
$$

$$
\begin{aligned}
\delta_{2}= & -\frac{1}{8 \sigma_{0}} \int \mathrm{d} z_{1} \int \mathrm{d} \mathbf{r}_{12} u^{\prime}(r) r\left(1-3 s^{2}\right)\left(z_{2}-z_{1}\right) \\
& \times \rho_{0}^{(2)}\left(z_{1}, z_{2}, r\right) .
\end{aligned}
$$

Both $\delta_{1}$ and $\delta_{2}$ were evaluated separately for each simulation of a planar liquid slab. In addition, Eqs. (34) and (35) were also evaluated using DFT replacing the pair density by the product of the densities. The results are summarized in Table $\mathrm{V}$. As seen in Table $\mathrm{V}, \delta_{1}$ and $\delta_{2}$ are both quite large, approximately equal in magnitude but of opposite sign. In this case, both $\delta_{1}$ and $\delta_{2}$ as determined via simulation are larger in magnitude than those determined via DFT. Their sum,

TABLE V. Comparison of values for the Tolman length for DFT and the simulation of the planar interface with $N=100000$.

\begin{tabular}{lclc}
\hline \hline & $\delta=\delta_{1}+\delta_{2}$ & \multicolumn{1}{c}{$\delta_{1}$} & $\delta_{2}$ \\
\hline DFT & -0.1462 & 2.0830 & -2.2292 \\
MD & 0.208 & 2.583 & -2.375 \\
\hline \hline
\end{tabular}


which equals the Tolman length, is quite sensitive to small differences in magnitude of both $\delta_{1}$ and $\delta_{2}$. The relative magnitudes of $\delta_{1}$ and $\delta_{2}$ suggests that the determination of $\delta_{1}$ via simulation is the origin of the sign discrepancy, though the reason for this is as yet unknown.

\section{CONCLUSION}

The Tolman length is an important quantity in the study of the excess free energy of a curved interface. However, there is much about it that is still not understood, even for simple fluids. ${ }^{3}$ The magnitude of the Tolman length has long been agreed upon to be $10 \%-20 \%$ of a molecular diameter. The sign of the Tolman length enjoys no such consensus. Calculations from DFT have always resulted in a negative Tolman length, ${ }^{4-8}$ while results from simulations have resulted in a positive Tolman length. ${ }^{9-11}$ These simulations have always been of a planar interface, taking advantage of the limit of infinite droplet size in order to determine the Tolman length. In this work, we focus instead on simulations of liquid droplets and determine the Tolman length from the Laplace equation, specifically, the dependence of the different in bulk pressures on the curvature. Two previous attempts to determine $\delta$ in this manner were inconclusive. ${ }^{12,13}$ Simulations of a hard-wall cavity in a liquid near coexistence by Moody and Attard ${ }^{14}$ resulted in a value of the Tolman length of -0.34 at $T^{*}=1$, which compares well to the value determined in this work. This value is also in reasonable agreement with the value of -0.29 determined from simulations based on a cluster model. ${ }^{15}$

In Eq. (21), we present a new expression for the difference in bulk pressure between a liquid droplet in coexistence with its vapor derived from expressions for the normal and tangential components of the Irving-Kirkwood pressure tensor. With this, we have two means of determining the difference in pressure. The first is to simply evaluate the components of the pressure tensor deep within the bulk phases. The second is Eq. (21), which uses an integral over the components of the pressure tensor throughout the interfacial region. These two methods agree well when the radius of the droplet is significantly large. For small droplet radii, the error bars become too large to conclude anything definite about the agreement between the two methods. By examining the dependence of $\Delta p$ on the curvature, we have shown that simulations of droplets result in a negative Tolman length, the first time such a result from simulation agrees with those from DFT.

The values of all physical properties in the extrapolated infinite-radius limit - except the Tolman length—are in complete agreement with those from the simulations of planar interfaces also presented in this work. To obtain the correct values for the physical properties of the planar interface, it was necessary to determine the (slight) dependence of said quantities on the system size. While this effect is subtle, it is nevertheless necessary for the comparison with the infiniteradius limit for the droplets.

One question in particular remains unanswered. Why does the Tolman length determined from the planar simulations via Eq. (6) not agree with that determined from the droplet simulations and from DFT? At present, the origin of this discrepancy is unclear. Several possibilities suggest themselves. (1) Eq. (6) is incorrect. However, the equivalent of Eq. (6) in DFT, Eqs. (29) and (31), are correct, so the expression itself does not seem to be at fault. (2) The implementation of Eq. (6) in the simulations is incorrect. Given that the sign and magnitude of $\delta_{1}$ and $\delta_{2}$ are consistent with DFT, this is also unlikely. Furthermore, a similar implementation is made for the evaluation of the virial-like expression for $\Delta p$ in Eq. (21) which is consistent with the direct evaluation of $\Delta p$. (3) The discrepancy is due to numerical inaccuracy. The length of the simulation runs and the sizes of the calculated standard distributions seem to preclude this. (4) The potential used in the simulations, Eq. (24), has a discontinuity in its first derivative at the cutoff $r=r_{\mathrm{c}}$. Sensitivity to this discontinuity might have a different influence in the different types of simulation. (5) The equimolar radius determined via Eq. (27) is an average value and as such is a constant. The instantaneous radius of the droplet, however, does fluctuate during the simulation with a magnitude of $\left(k_{\mathrm{B}} T / 4 \pi \sigma\right)^{1 / 2} \simeq 0.6$ at $T^{*}=0.9$, which is quite substantial compared to the magnitude of the Tolman length. Somehow, these capillary-wave fluctuations might be subtly responsible for the sign discrepancy.

\section{APPENDIX: DFT EXPRESSIONS FOR THE TOLMAN LENGTH}

Here, we list the results of DFT. The starting expression for the free energy is the usual expression

$$
\begin{aligned}
\Omega[\rho]= & \int \mathrm{d} \mathbf{r}\left[f_{\mathrm{hs}}(\rho)-\mu \rho(\mathbf{r})\right] \\
& +\frac{1}{2} \int \mathrm{d}_{1} \int \mathrm{d} \mathbf{r}_{2} u_{\mathrm{att}}(r) \rho\left(\mathbf{r}_{1}\right) \rho\left(\mathbf{r}_{2}\right) .
\end{aligned}
$$

For $f_{\text {hs }}(\rho)$ we take the well-known Carnahan-Starling form ${ }^{24}$

$$
f_{\mathrm{hs}}(\rho)=k_{\mathrm{B}} T \rho \ln (\rho)+k_{\mathrm{B}} T \rho \frac{\left(4 \eta-3 \eta^{2}\right)}{(1-\eta)^{2}},
$$

where $\eta \equiv(\pi / 6) \rho \sigma^{3}$ with $\sigma$ the molecular diameter (not to be confused with $\sigma_{0}$, the surface tension of a planar interface).

For the attractive part of the interaction potential $u_{\text {att }}(r)$ [see Eq. (24)], we take

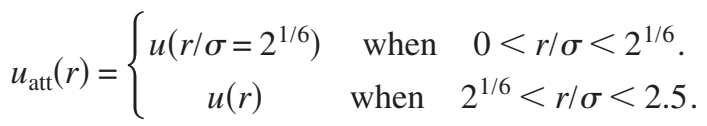

This means that $u_{\text {att }}(r)$ is a continuous function with the benefit that $u_{\text {att }}^{\prime}(r)$ has no delta-function contributions that have to be taken into account separately.

With this expression for $u_{\text {att }}(r)$, the bulk phase diagram can be determined from $f(\rho)=f_{\text {hs }}(\rho)-a \rho^{2}$ where the van der Waals parameter $a$ can be explicitly expressed in terms of the reduced temperature variable $T^{*} \equiv k_{\mathrm{B}} T / \varepsilon$ using 


$$
a \equiv-\frac{1}{2} \int \mathrm{d} \mathbf{r}_{12} u_{\mathrm{att}}(r)
$$

To determine the position in the phase diagram in the DFT calculations, we could simply set $T^{*}=0.9$ and determine $a$ from the expression above. Here, we choose the somewhat more common method to determine the position in the phase diagram by matching the density difference $\Delta \rho=\rho_{\ell}-\rho_{v}$ to the value obtained in the simulations. In Table IV, we list the various quantities obtained from the simulations and from DFT.

The Euler-Lagrange equation that minimizes the free energy in Eq. (A1) is

$$
f_{\mathrm{hs}}^{\prime}(\rho)=\mu-\int \mathrm{d} \mathbf{r}_{12} u_{\mathrm{att}}(r) \rho\left(\mathbf{r}_{2}\right)
$$

When we consider the free energy of a liquid droplet and expand the Euler-Lagrange equation in its radius $1 / R$, one finds that $\rho_{0}(z)$ and $\rho_{1}(z)$ are determined by

$$
\begin{aligned}
f_{\mathrm{hs}}^{\prime}\left(\rho_{0}\right)=-\int \mathrm{d} \mathbf{r}_{12} u_{\mathrm{att}}(r) \rho_{0}\left(z_{2}\right), \\
\begin{aligned}
f_{\mathrm{hs}}^{\prime \prime}\left(\rho_{0}\right) \rho_{1}\left(z_{1}\right)= & \mu_{1}-\int \mathrm{d} \mathbf{r}_{12} u_{\mathrm{att}}(r) \\
\times & {\left[\rho_{1}\left(z_{2}\right)+\frac{1}{2} r^{2}\left(1-s^{2}\right) \rho_{0}^{\prime}\left(z_{2}\right)\right] . }
\end{aligned}
\end{aligned}
$$

These two equations are used to expand the free energy in Eq. (A1) in $1 / R$ and identify the surface tension

$$
\sigma_{0}=-\frac{1}{4} \int_{-\infty}^{\infty} \mathrm{d} z_{1} \int \mathrm{d} \mathbf{r}_{12} u_{\mathrm{att}}(r) r^{2}\left(1-s^{2}\right) \rho_{0}^{\prime}\left(z_{1}\right) \rho_{0}^{\prime}\left(z_{2}\right)
$$

and Tolman length, for which we have two expressions,

$$
\delta=\frac{1}{4 \sigma_{0}} \int_{-\infty}^{\infty} \mathrm{d} z_{1} \int \mathrm{d} \mathbf{r}_{12} u_{\mathrm{att}}(r) r^{2}\left(1-s^{2}\right) z_{1} \rho_{0}^{\prime}\left(z_{1}\right) \rho_{0}^{\prime}\left(z_{2}\right),
$$

$$
\delta=\frac{1}{4 \sigma_{0}} \int_{-\infty}^{\infty} \mathrm{d} z_{1} \int \mathrm{d} \mathbf{r}_{12} u_{\mathrm{att}}(r) r^{2}\left(1-s^{2}\right) \rho_{0}^{\prime}\left(z_{1}\right) \rho_{1}^{\prime}\left(z_{2}\right) .
$$

It is straightforward to show the equivalence of Eq. (A9) to Eq. (A10) by using the Euler-Lagrange equation for $\rho_{1}(z)$ in Eq. (A7).

${ }^{1}$ J. S. Rowlinson and B. Widom, Molecular Theory of Capillarity (Clarendon, Oxford, 1984).

${ }^{2}$ R. Tolman, J. Chem. Phys. 17, 333 (1949).

${ }^{3}$ E. M. Blokhuis and J. Kuipers, J. Chem. Phys. 124, 074701 (2006).

${ }^{4}$ V. Talanquer and D. W. Oxtoby, J. Phys. Chem. 99, 2865 (1995).

${ }^{5}$ A. E. van Giessen, E. M. Blokhuis, and D. J. Bukman, J. Chem. Phys. 108, 1148 (1998).

${ }^{6}$ T. V. Bykov and X. C. Zeng, J. Chem. Phys. 111, 3705 (1999).

${ }^{7}$ J. Barrett, J. Chem. Phys. 111, 5938 (1999).

${ }^{8}$ J. C. Barrett, J. Chem. Phys. 124, 144705 (2006).

${ }^{9}$ A. E. van Giessen and E. M. Blokhuis, J. Chem. Phys. 116, 302 (2002).

${ }^{10}$ M. J. Haye and C. Bruin, J. Chem. Phys. 100, 556 (1994).

${ }^{11}$ P. R. ten Wolde and D. Frenkel, J. Chem. Phys. 109, 9901 (1998).

${ }^{12}$ M. J. P. Nijmeijer, C. Bruin, A. B. van Woerkom, A. F. Bakker, and J. M. J. van Leeuwen, J. Chem. Phys. 96, 565 (1992).

${ }^{13}$ S. M. Thompson, K. E. Gubbins, J. P. R. B. Walton, R. A. R. Chantry, and J. S. Rowlinson, J. Chem. Phys. 81, 530 (1984).

${ }^{14}$ M. P. Moody and P. Attard, J. Chem. Phys. 115, 8967 (2001).

${ }^{15}$ D. I. Zhukhovitskii, Russ. J. Phys. Chem. 75, 1043 (2001).

${ }^{16}$ E. M. Blokhuis and D. Bedeaux, Physica A 184, 42 (1992); J. Chem. Phys. 95, 6986 (1991); Mol. Phys. 80, 705 (1993); Heterog. Chem. Rev. 1, 55 (1994).

${ }^{17}$ J. H. Irving and J. G. Kirkwood, J. Chem. Phys. 18, 817 (1950).

${ }^{18}$ E. M. Blokhuis and D. Bedeaux, J. Chem. Phys. 97, 3576 (1992).

${ }^{19}$ C. D. Holcomb, P. Clancy, S. M. Thompson, and J. A. Zollweg, Fluid Phase Equilib. 75, 185 (1992); C. D. Holcomb, P. Clancy, and J. A. Zollweg, Mol. Phys. 78, 437 (1993); E. M. Blokhuis, D. Bedeaux, C. D. Holcomb, and J. A. Zollweg, ibid. 85, 665 (1995).

${ }^{20}$ D. Frenkel and B. Smit, Understanding Molecular Simulations (Academic, San Diego, 2002).

${ }^{21}$ F. Biscay, A. Ghoufi, F. Goujon, V. Lachet, and P. Malfreyt, J. Chem. Phys. 130, 184710 (2009).

${ }^{22}$ H. El Bardouni, M. Mareschal, R. Lovett, and M. Baus, J. Chem. Phys. 113, 9804 (2000).

${ }^{23}$ E. M. Blokhuis and J. Kuipers, J. Chem. Phys. 126, 054702 (2007).

${ }^{24}$ N. F. Carnahan and K. E. Starling, Phys. Rev. A 1, 1672 (1970). 